\title{
Design Considerations and Experimental Studies on Semi-Active Smart Pin Joint
}

\author{
Yancheng LI, Jianchun LI, Bijan SAMALI \\ Centre for Built Infrastructure Research, School of Civil and Environmental Engineering, Faculty \\ of Engineering and Information Technology, University of Technology Sydney, NSW 2007, \\ Australia \\ Jiong WANG \\ School of Mechanical Engineering, Nanjing University of Science and Technology, Nanjing, \\ Jiangsu 210094, China
}

\begin{abstract}
Hostile dynamic loadings such as severe wind storms, earthquakes, and sudden impacts can cause severe damage to many civil engineering structures. An intelligent structural system equipped with smart structural members that are controllable in real-time is one effective solution to prevent structural damage and failure during such situations. Civil intelligent structures with controllable properties to adapt to any changes due to dynamic loadings can lead to effective protection of structures and their occupants. In this paper, design and testing of a semi-active magnetorheological (MR) pin joint, in which the moment resistance can be controlled in real-time by altering the magnetic field, is reported with the view to be used as a potential candidate for smart members in development of intelligent structures. As a preliminary research aimed at full-development of an intelligent structure, the primary purpose of the current study is to design, fabricate and characterise a prototype smart member, namely a semi-active MR pin joint, through theoretical modeling and experimental testing. Design of prototype smart pin joints includes theoretical analysis relating to the radius of rotary plate, the property of MR fluids and the gap between the rotary plate and the casing based on the requirements of the dynamics of MR pin joints. FEM analysis was deployed to study the distribution of the magnetic field along the gap. It is found, from the theoretical analysis and experimental verification, that the MR pin joint with a diameter of $180 \mathrm{~mm}$ can produce a torque of up to $30 \mathrm{Nm}$, which meets requirements as semi-active members in a multi-storey prototype building model in the next stage of research and development.
\end{abstract}

Key words: Magnetorheological Fluids, Smart Pin Joint, Design Consideration;

\section{Introduction}

Magnetorheological (MR) fluid is a kind of rheology-controllable fluid whose rheological state can be altered from Newtonian fluid to gel-like solid in few milliseconds when subjected to a magnetic field [1-2]. It consists of three parts, which are: microscopic magnetic iron particles, carrier liquid and the additives. The carrier fluids can be silicone oil, hydrocarbon oil or water depending on the specific application. Having a size range of $0.1-10 \mu \mathrm{m}$, the magnetic particles usually scatter 
freely in the carrier liquid in normal state and form a chain structure along the magnetic flux when magnetized [3]. These chain-like structures restrict the motion of the fluid, thereby increasing the viscous characteristics of the suspension. The mechanical energy needed to yield these chain-like structures increases as the applied magnetic field increases resulting in a field dependent yield stress. The yield stress of the MR fluid can be up to $100 \mathrm{kPa}$. This process, namely, MR effect, is a repeatable and reversible effect and can be accurately controlled by varying the density of the magnetic field.

MR fluid can be used to develop high-capacity semi-active devices due to its low power requirement and real-time controllability. There have been increasing attentions on bringing this technology out of the laboratory and into industry because of its great potential for use in automotive applications. Commercial MR products such as MR fluid damper, MR brake, etc, have been prototyped to be implemented in the automobile suspension, seat suspension and heavy trucks*. Gordaninejad et al [4] designed, developed and tested a new MR fluid damper for high-mobility multi-purpose wheeled vehicle. Wereley [5] developed MR fluid damper for the rotation system of a helicopter. With respect to applications in civil engineering, Spencer et al [6] built a 20 ton large-scale MRF damper and piloted a phenomenal model using Bouc-Wen Hysteresis model. Ni [7] successfully implemented the MR fluid damper on the stayed-cable bridge of Dongting Lake in China, to reduce the vibration caused by the wind and the rains. However, in comparison with the rapid and vast developments in mechanical engineering and aeronautical engineering, research and development in the use of MR fluid in civil engineering has been very limited.

On the other hand, mitigating the effects from severe dynamic loadings, such as earthquakes, strong winds, as well as sudden impacts and explosions, has always been a challenge for civil engineers [8]. As most civil engineering structures have fixed natural frequencies, the effects of dynamic loading will be greatly amplified when the structural natural frequencies fall within the range of excitation frequencies. It is, therefore, highly desirable if a control strategy can be implemented to shift the frequencies of civil engineering structure away from resonance inducing excitations in real time to mitigate harmful dynamic loadings [9].

In this paper, a new semi-active structural member utilizing MR fluid, namely, MR pin joint, is proposed to be an effective candidate. A smart MR pin joint is a smart structural member whose joint moment resistance can be controlled in real-time by altering the magnetic field. The smart pin joint is used as a rotational connector at the column-beam connection in a civil structure. By changing the current in the magnetic coils, the output torque of the MR pin joint can be adjusted from nearly zero joint moment (pinned) to full capability (locked). Hence, the rigidity of the structural connection can be controlled from fully rigid to fully pinned as well as any state in between. Thereby, the natural frequencies of civil structures can be shifted in order to avoid the resonance region and therefore prevent the structure from unwanted damage. 


\section{Semi-Active MR Pin Joint}

A sketch of a disk-type MR pin joint is shown in Figure 1. The MR pin joint mainly consists of five parts: (1) a rotary thin plate inside the pin joint, (2) a shaft connected to the plate to transfer the joint moment, (3) two uniform housings to form the hollow cavity, (4) MR fluids between the housing and plate, and (5) a circular coil producing magnetic field to magnetize MR fluids. There are gaps of about $1 \mathrm{~mm}$ between the plate and the housings, to hold the MR fluids therein. Inside the gap is filled with MR liquid (MRF140CG) from Lord Corporation, which can provide the yield stress of up to $60 \mathrm{kPa}$ at saturated magnetic field of $\mathrm{B}=1.0 \mathrm{~T}$. Due to the high capacity of MR fluids, this MR pin joint can produce about $30 \mathrm{Nm}$ with a saturated current of $2.0 \mathrm{~A}$, as shown in part 4 in this paper. Other structural parameters of the pin joint are listed in Table 1.

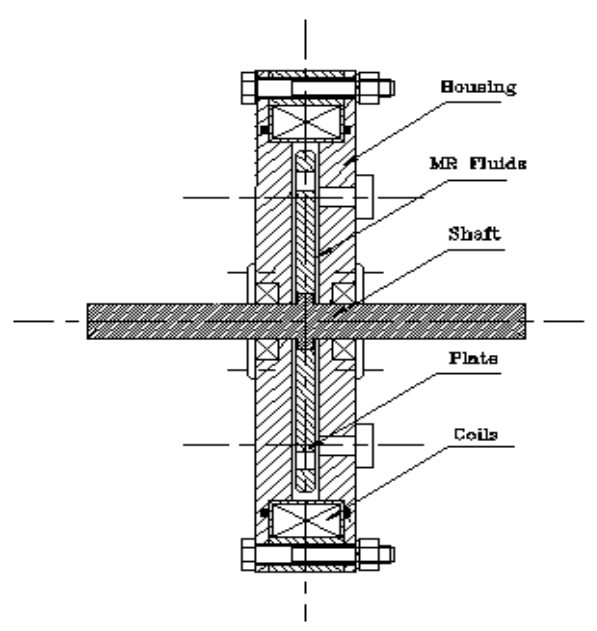

Figure 1. Sketch map of MR pin joint

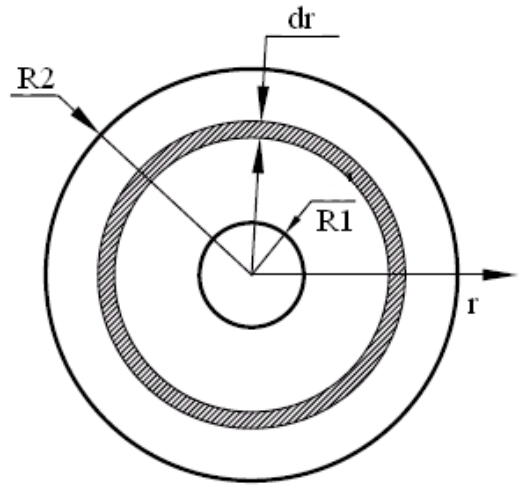

Figure 2. Rotary plate under shear stress

Table 1. Structural parameters of the MR pin joint (mm)

\begin{tabular}{llll}
\hline Shaft radius $\mathbf{R}_{\mathbf{1}}$ & 12.5 & Outer diameter $\mathbf{D}$ & 180 \\
\hline Radius of rotary plate $\mathbf{R}_{\mathbf{2}}$ & 50 & Inner diameter $\mathbf{d}$ & 120 \\
\hline Gap $\mathbf{h}$ & 1 & Thickness $\mathbf{B}$ & 70 \\
\hline
\end{tabular}

\section{Theoretical Modeling of Semi-Active MR Pin Joint}

Inside a MR pin joint, the MR fluids flow rotationally between the plate and the housing, as shown in Figure 2. Compared with the plate diameter (usually 0.5-1.0 $\mathrm{mm}$ ), the gap between the plate and the housing is very small. Thus, the flow of MR fluids in the gap can be regarded as the flow between two parallel plates.

For any micro-body in the plate, the area of body is

$$
d S=2 \pi r \cdot d r
$$

$$
d T=F \cdot r=(\tau \cdot d S) \cdot r=\tau \cdot 2 \pi r^{2} \cdot d r
$$


Then the torque applied on one side of the plate is

$$
T_{1}=\int d T=\int_{R_{1}}^{R_{2}} \tau \cdot 2 \pi r^{2} \cdot d r
$$

As a kind of non-newtonian fluid, the behavior of the MR fluid is often represented as a Bingham plastic model having variable yield strength. In this model, the flow is governed by Bingham's equations:

$$
\tau=\tau_{y}(B)+\eta \frac{\partial u}{\partial r} \quad \tau \geq \tau_{\mathrm{y}}
$$

at the stresses $\tau$ above the field dependent yield stress $\tau_{y}$. Where $\tau$ is the shear stress, $\tau_{y}$ is the dynamic yield stress, $\eta$ is the fluids viscosity, and $u$ the velocity of the plate.

Below the yield stress, the material behaves viscoelastically:

$$
\tau=G \gamma \quad \tau<\tau_{\mathrm{y}}
$$

Where $\mathrm{G}$ is the complex material modulus. Bingham plastic model has proven useful in the design and characterisation of MR fluid-based devices.

For disc-like MR pin, the Bingham plastic model can be rewritten as

$$
\begin{array}{rlrl}
\tau & =\tau_{y}(B)+\eta \frac{r \cdot \omega}{h} & & \tau \geq \tau_{\mathrm{y}} \\
\tau & =G \gamma & \tau<\tau_{\mathrm{y}}
\end{array}
$$

Where, $\omega$ is the angular velocity of the plate, $r$ is the position in the plate surface (shown in figure 2), $h$ is the gap size between the plate the housing. It should be noted here that the yield stress $\tau_{y}$ is the function of magnetic field density B. Changing the current in the coil will result in the variety of magnetic field density.

In this analysis, we assume that the MR fluids work in post-yield region. Therefore, the total torque on the MR pin joint is

$$
\begin{gathered}
T=2 \int d T=2 \int_{R_{1}}^{R_{2}}\left(\tau_{y}+\eta \frac{r \cdot \omega}{h}\right) \cdot 2 \pi r^{2} \cdot d r \\
T=\frac{\pi \eta \omega}{h}\left(R_{2}^{4}-R_{1}^{4}\right)+\frac{4}{3} \pi\left(R_{2}^{3}-R_{1}^{3}\right) \tau_{y}
\end{gathered}
$$

The torque that a MR brake produces consists of two parts:

$$
\begin{gathered}
T_{\eta}=\frac{4}{3} \pi\left(R_{2}^{3}-R_{1}^{3}\right) \tau_{y} \\
T_{v i s}=\frac{\pi \eta \omega}{h}\left(R_{2}^{4}-R_{1}^{4}\right)
\end{gathered}
$$


Where, coulomb torque, $T_{\eta}$, is the torque related to the yield stress of MR fluids due to coil current; Viscosity torque, $\mathrm{T}_{\text {vis}}$, is the torque related to the fluid viscosity. Hence, the total torque produced by the MR pin joint is:

$$
T_{\text {total }}=T_{\eta}+T_{\text {vis }}+T_{\text {fri }}
$$

Where, $T_{\text {fri }}$ is the friction torque induced by the friction between the outer housing and the shaft. It should be noted that the friction torque is very small compared to the total torque and will not be considered in theoretical analyses.

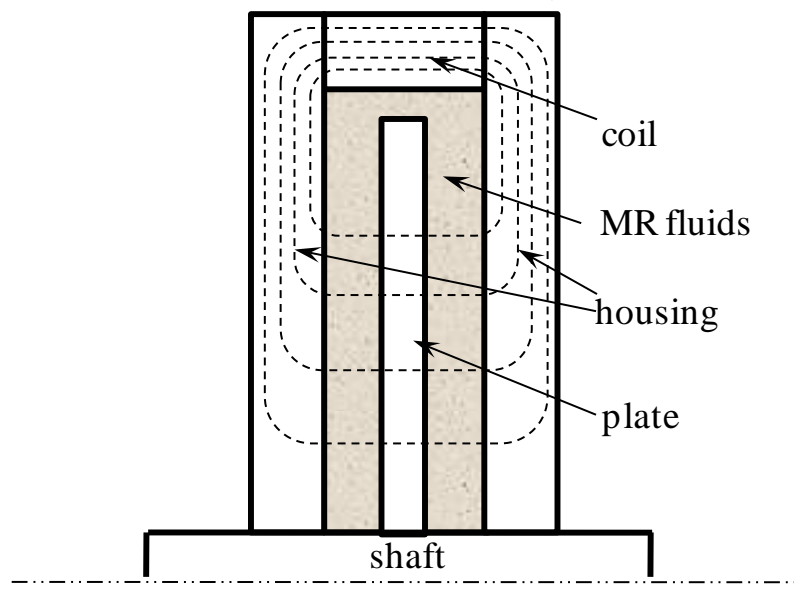

Figure 3. Magnetic circuit in the MR pin

"Dr. Dave's" empirical equation [10] shows the inerratic relation of the yield stress of the MR fluids, the magnetic field density and magnetic field intensity, as following:

$$
\begin{gathered}
\tau_{M R}=C \cdot 271700 \cdot \phi^{1.5239} \cdot \tanh \left(6.33 \times 10^{-6} \cdot H\right) \\
B=1.91 \cdot \phi^{1.133} \cdot\left[1-\exp \left(-10.97 \cdot \mu_{0} \cdot H\right)\right]+\mu_{0} \cdot H
\end{gathered}
$$

In which, $\Phi$ is the volume fraction of iron particles in the MR fluids, $\mu_{0}$ is the magnetic constant, $\mu_{0}=4 \pi \times 10^{-7}$, C equals $1.0,1.16$, or 0.95 depending on whether the carrier fluid is hydrocarbon oil, water or silicone oil. For MRF140CG, C=0.95.

Following is the analysis on magnetic circuits in order to explore the relationship of controllable yield stress and the coil current. Inside the MR pin, the coil, housing, rotational plate and MR fluids form a closed-loop magnetic circuit, as shown in figure 3. Maxwell-Ampère's Circuital Law relates the magnetic field to its electric current source as following:

$$
\oint_{L} H \cdot d l=\sum i=N \cdot I
$$

Where, $H$ is the magnetic field intensity, $L$ is the magnetic circuit, $I$ is the operating current penetrating through the coil, $N$ is the turn of the coil. In this design, $\mathrm{N}=140$.

The relationship between the field-dependent yield stress of MR fluid and the operating current can be concluded by combining equation (3), (6), (7) and (8). 


\section{Design considerations}

\subsection{Viscosity Torque and Coulomb Torque}

Recalling equations (4) and (5), the overall torque includes viscosity torque $T_{\text {vis }}$ and Coulomb torque $\mathrm{T}_{\eta}$. Figure 4 indicates the distribution of these two types of torques from the theoretical simulation when the coil current is 1.0A.

It is clear that the Coulomb torque is the main contributor to the total torque of the MR pin since viscosity torque is really small, e.g. when the viscosity torque is about $1 \mathrm{Nm}$ the Coulomb torque is more than $20 \mathrm{Nm}$. It can also be observed that the rotary speed has little influence on the total torque. The effect of rotary speed on the torque output can numerically be shown by Figure 5 . By increasing rotary speed from 0 to $240 \mathrm{rpm}$, the torque is increased by only $0.171 \mathrm{Nm}$.
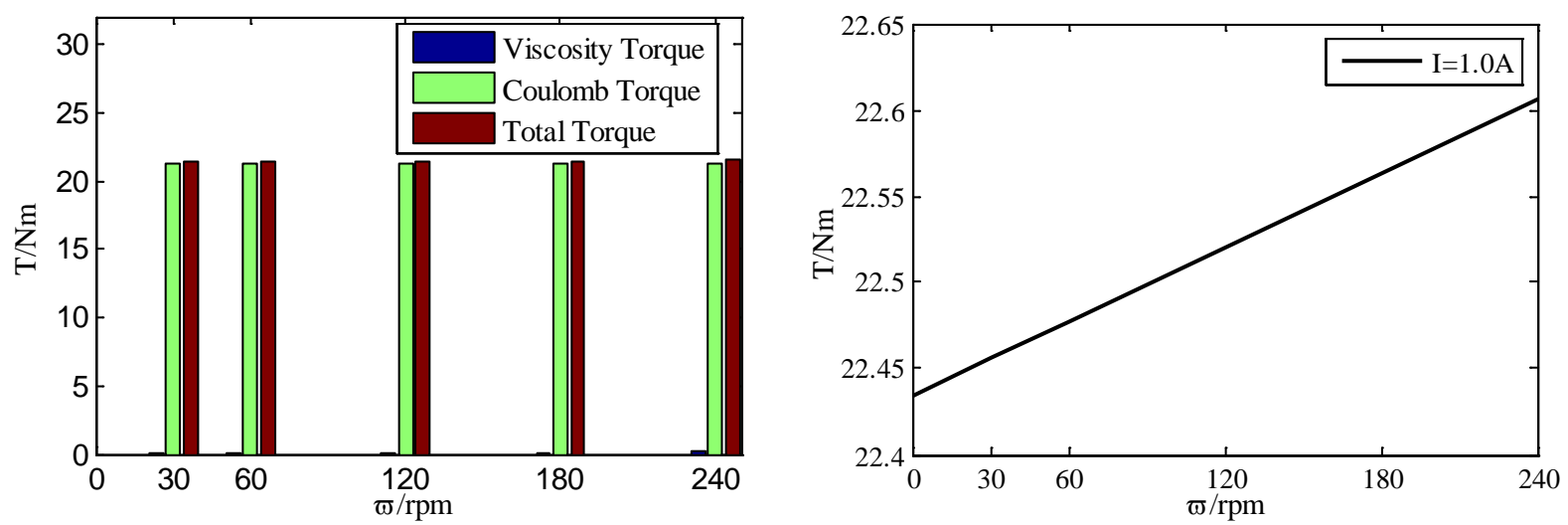

Figure4. Distribution of viscosity torque and total torque ( $\mathrm{I}=1 \mathrm{~A})$ Figure 5. Impact of rotary speed on total torque

\subsection{MR Device Parametric Analysis}

To investigate the impact of device parameters such as the gap, shaft radius and plate radius on the total torque output, numerical simulations were conducted.

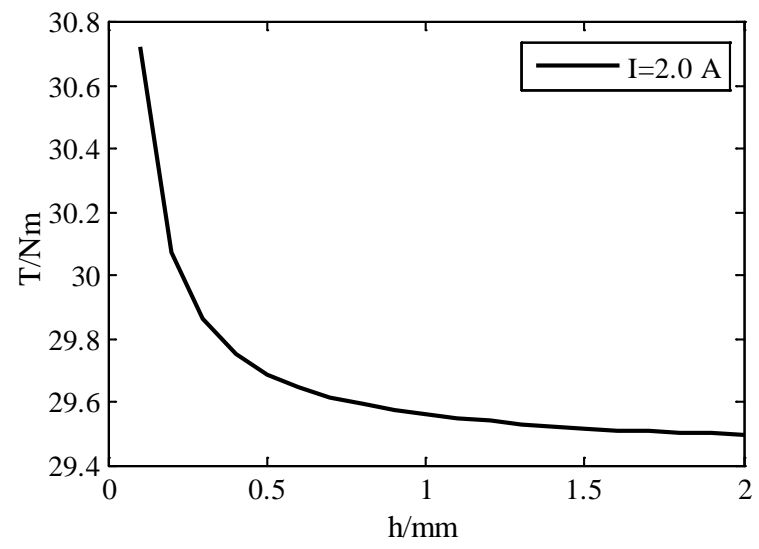

Figure 6. Impact of gap dimension on total torque

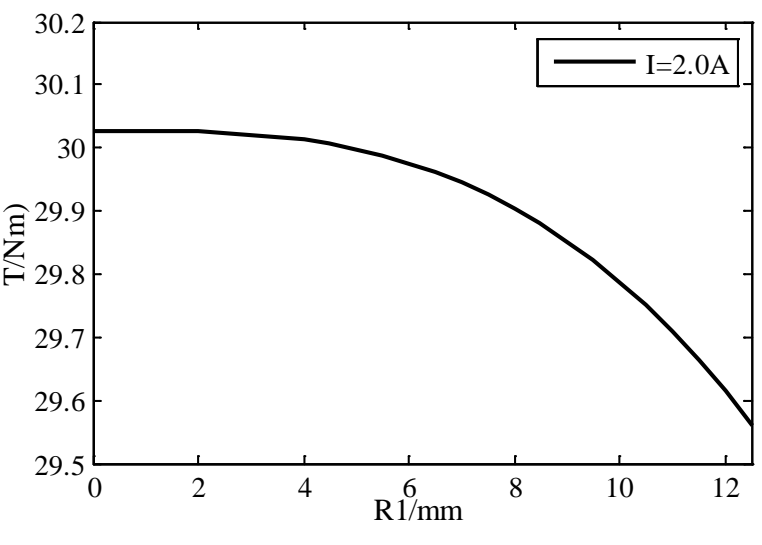

Figure 7. Impact of shaft radius on total torque

Figure 6 shows the effect of gap dimension on the capacity of MR pin joint at the current level of 2.0A. Unlike the MR damper in which the gap size is critical for its 
damping ability, it is of less importance in MR pin joint. Since the gap should not be smaller than $0.5 \mathrm{~mm}$ in real applications due to increased difficulty in fabrication and operation, a relatively larger gap is acceptable in designing a MR pin joint.

The impact of shaft radius and plate radius on the torque are shown in Figures 7 and 8 , respectively. The plate radius is of critical importance among the structural parameters in development of a large-torque MR pin joint from the analysis. For example, a small plate radius less than $35 \mathrm{~mm}$ leads to a torque capacity of no more than $10 \mathrm{Nm}$.

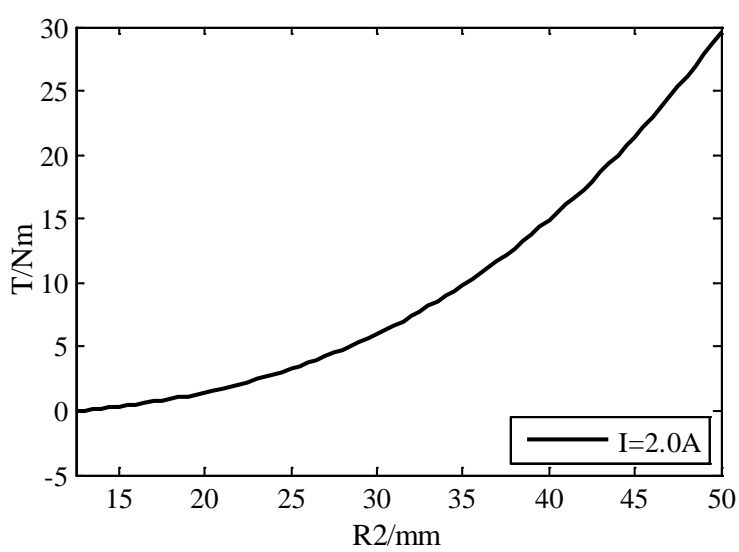

Figure 8. Impact of plate radius on total torque

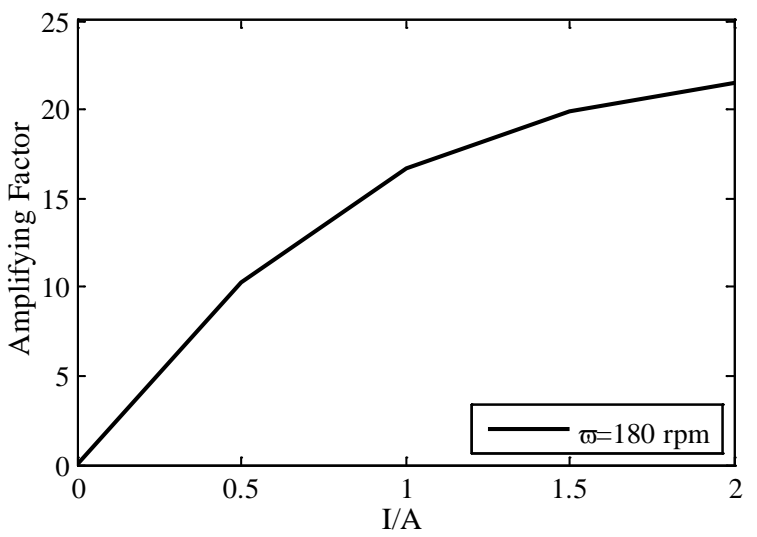

Figure 9. Amplifying factor vs. current

\subsection{Amplifying Factor}

An amplifying factor is introduced in this section to investigate the dynamic performance of the MR pin joint. The amplifying factor $\alpha$ is defined as the ratio of the total torque output vs. the viscosity torque, that is:

$$
\alpha=\frac{T_{\text {field on }}}{T_{\text {field off }}}=\frac{T_{\text {total }}}{T_{\text {vis }}}
$$

A plot of the amplifying factor vs. current at the rotary speed of $180 \mathrm{rpm}$ is shown in Figure 9. With increasing coil current, the amplifying factor increases from 1 to nearly 22. This indicates that the higher the coil current is, the more control freedom the MR pin joint has.

\subsection{FEM Analysis of the Magnetic Circuits}

The key issue in designing a MR pin joint is to ensure that the magnetic field through the gap is at least equal to the saturated field of the MR fluids. In other words, the magnetic field through the MR fluids should be maintained constant and saturated. To achieve a saturated magnetic field in the gap, the magnetic properties of the housings, rotary plate and shaft must be carefully considered during the structural design process. The MR rotary pin joints require highly magnetically permeable pole pieces for directing the magnetic flux across the working fluid gaps contained therein. In some cases there can be unwanted stray magnetic flux which can detract from the 
effectiveness of the device. Stray or flanking flux paths can lead to a loss of efficiency because magnetic fields and associated field energy are developed where they are not needed or desired [11]. This may cause remnant magnetism in any hard-magnetizable component present in the pin joint. This remnant magnetism can attract the magnetically-soft medium into areas where it may be undesirable to have it, for example, adjacent to the shaft seal.

Since the magnetic properties of the MR fluids and the medium carbon steel used are typically nonlinear, it is not reliable to accurately determine the magnetic fields by theoretical analysis. In this section, a 2D FEM analysis is carried out using Maxwell Ansoft software. Table 2 lists the materials of every part in MR pin joint.

Table 2. Materials of every part

\begin{tabular}{llll}
\hline Part & Material & Part & Material \\
\hline Magnetic coil & Copper & Plate & Medium carbon steel \\
Shaft & Medium carbon steel & Coil casing & Plastic \\
Outer housing & Medium carbon steel & & \\
\hline
\end{tabular}

Since MR pin joint is a typical centrosymmetric device, a $1 / 2$ section plane is adopted in FEM analysis. Figure 10 shows the magnetic fields density along the radial direction at a current of 1.0A. It can be seen from the FEM analysis that the magnetic field density in the shaft and outer housing is far higher than that in the MR fluids. Especially, the magnetic field density in the shaft is the highest inside the MR pin. It is because the relative magnetic permeability of the MR fluids is far smaller than that of medium carbon steel used to make up the shaft and outer housing. However, the magnetic densities in MR fluids and the plate are almost the same.

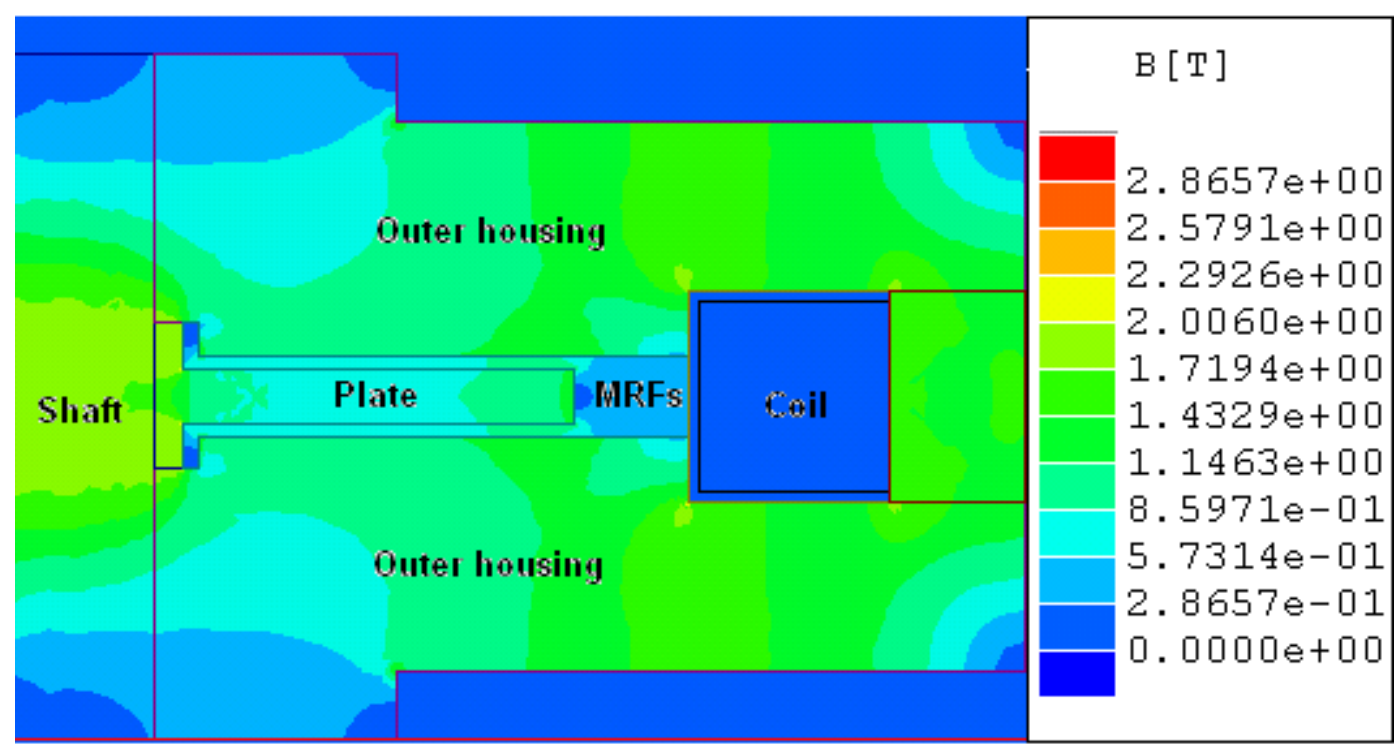

Figure 10. Magnetic field density in MR pin joint (1.0 A)

A quantitative analysis along the plate surface and the housing surface at the current of 1.0A and 2.0A are listed in Figure 11. Same conclusions as to the magnetic field density gap derived from Figure 10 can also be drawn in Figure 11. Furthermore, the 
magnetic density along the plate surface increases from $0.7 \mathrm{~T}$ to $1.0 \mathrm{~T}$ when the coil current increases from 1.0A to 2.0A. Since the critical magnetic saturation field of MRF140CG is 1.0T, it is clear that 2.0A is the critical current for the MR pin joint, which means that the magnetic field density will not increase distinctly when the coil current is above 2.0A. The finite element analysis also validates the theoretical estimation in which the predicted saturated current is about 2.0A.
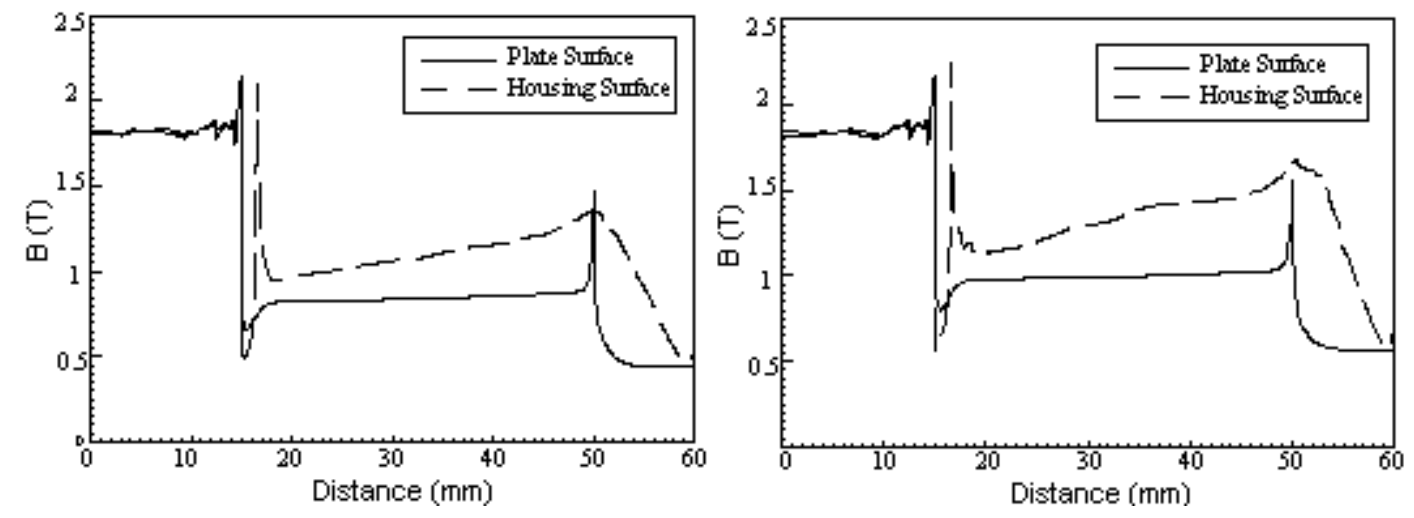

Figure 11. Magnetic field density along the plate surface and housing surface (1.0A and 2.0 A)

\section{Experimental Set-Up}

To validate the design and evaluate the performance of the MR pin joint, an experiment was conducted and its set-up is as shown in Figure 12. The set-up consists mainly of four parts: (1) an AC motor, (2) a torque sensor, (3) a DC power supply and (4) the MR pin joint. The AC motor produces a series of rotary motions at speeds ranging from 0 to $500 \mathrm{rpm}$ achieved by an adjustable frequency inverter (SA2150). A torque sensor (JN338) with a capacity of $50 \mathrm{Nm}$, was used to measure the torque transmitted from the MR pin joint to AC motor. The AC motor, torque sensor and the MR pin joint were installed in such a way that all the centroids were aligned along the same horizontal line. A DC power supply provided the required currents to the magnetic coil in the MR pin joint. A multi-meter was connected to the DC power supply and the MR pin joint to measure the required current.

Two connectors were implemented between the AC motor and torque sensor, and the torque sensor and the MR pin joint to transmit the torque to each other. The test rig was fixed to solid ground in order to reduce unwanted vibrations. A series of tests at different rotary speeds, namely, $30 \mathrm{rpm}, 60 \mathrm{rpm}, 120 \mathrm{rpm}, 180 \mathrm{rpm}$ and $240 \mathrm{rpm}$, were conducted to investigate the static performance of MR pin joint. During the tests, all data were recorded after AC motor output a steady rotary speed.

\section{Experimental Results}

Five rotary speeds, namely, 30, 60, 120, 180 and $240 \mathrm{rpm}$, corresponding to the rotary frequencies of $0.5,1,2,3$ and $4 \mathrm{~Hz}$, were used in the test. Moreover, for MR pin joints five coil currents, i.e., 0, 0.5, 1.0, 1.5 and 2.0 Amp, were introduced using the DC power supply. 
Figure 13 shows a plot of the transmitted torque versus the coil current of the MR pins at various rotary speeds of $\omega=30 \mathrm{rpm}, 60 \mathrm{rpm}, 120 \mathrm{rpm}, 180 \mathrm{rpm}$ and $240 \mathrm{rpm}$. It can be seen from the figure that the torque is not sensitive to different rotary speeds. In other words, the coil current can be considered as the sole factor for torque output of MR pins in an approximate model.

Figure 14 shows a series of transmitted torques versus rotary speeds at five current levels, i.e. I=0 A, 0.5 A, 1.0 A, 1.5 A and 2.0 A. Once again, it can be observed from Figure 13 that in the steady state output torques are nearly independent of the rotary speed. For example, with the increase of rotary speed from $30 \mathrm{rpm}$ to $240 \mathrm{rpm}$ at the current of $0.5 \mathrm{~A}$, the torque increases insignificantly from $12.84 \mathrm{Nm}$ to $13.53 \mathrm{Nm}$. Figure 14 can also validate the design, for example, the saturated current of the MR pin joint is about 2.0 Amp which is consistent with the theoretical estimation.

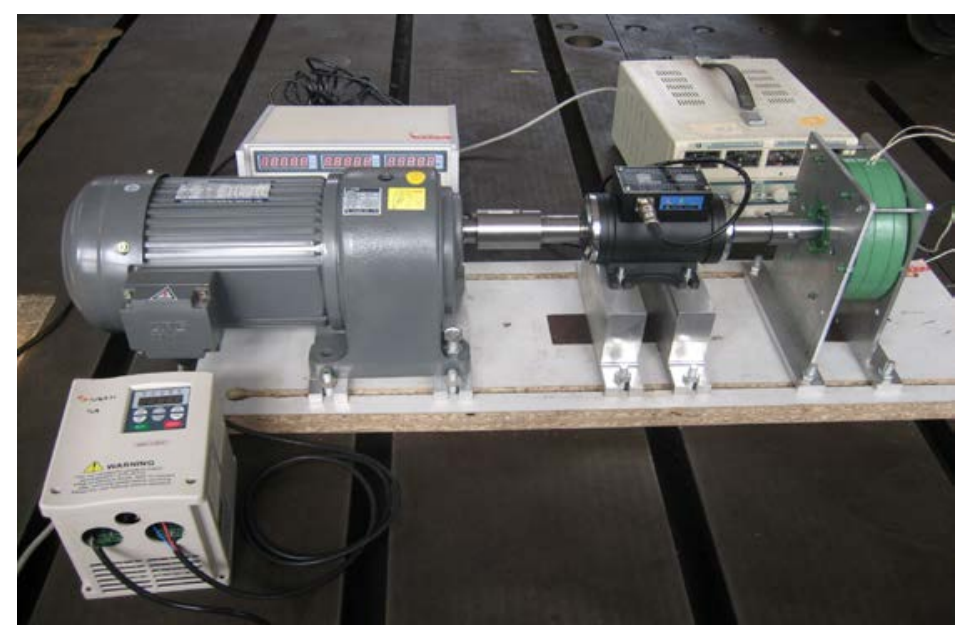

Figure 12 Experimental set-up of MR pin joint test

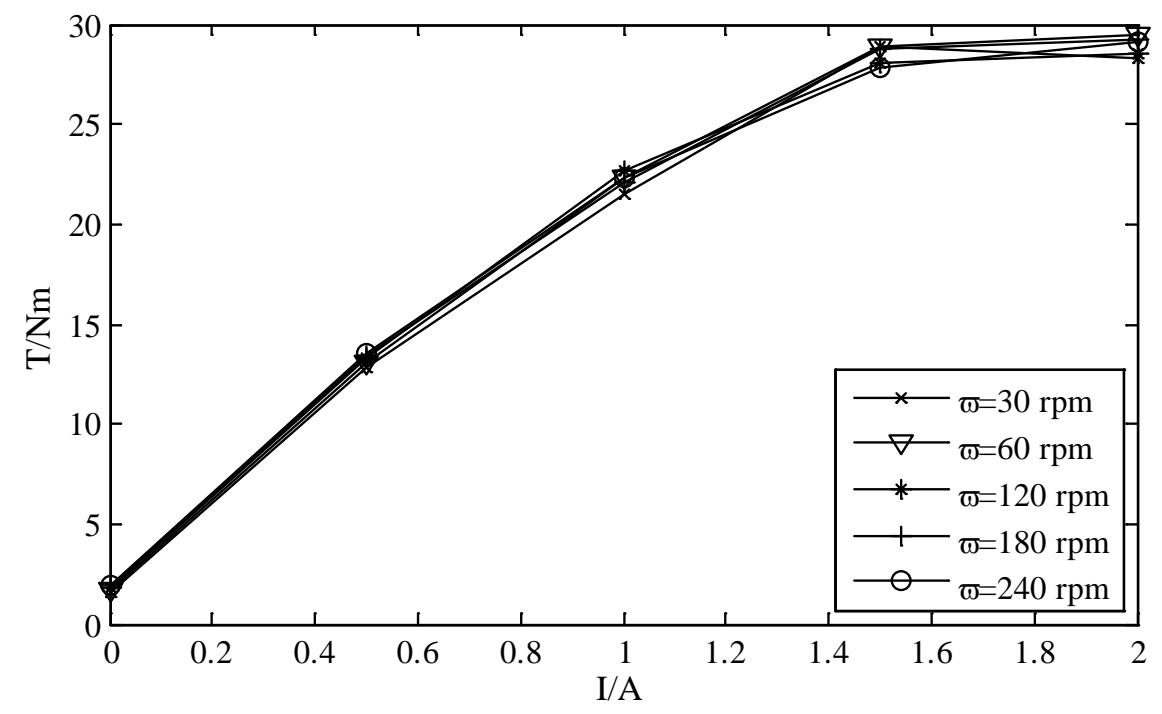

Figure 13 Total transmitted torque vs. coil current at different rotary speeds

A comparison between theoretical analysis and experimental results is shown in Figure 15. It is seen that the theoretical analysis and the experimental results match very well. The distinct lag between the theoretical results and the experimental curve 
is due to the fact that the friction torque is not considered in the theoretical analysis. It can also be observed that a linear relationship exists between the total torque and the coil current before the saturation point.

Conclusively, both theoretical analysis and experimental tests show that the designed MR joint possesses an ability to dramatically change the output moment resistance in a controllable manner. For example, the torque of the MR pin joint can be changed from less than $1 \mathrm{Nm}$ to $30 \mathrm{Nm}$. Regardless of the rotary speed, the capacity of MR pin joint can be easily controlled by altering the current in the magnetic coil. Possessing a small zero-field torque and large saturated torque, the designed MR pin joint can be considered a good candidate for smart members to realize the structural frequency shifting of the intelligent structure.

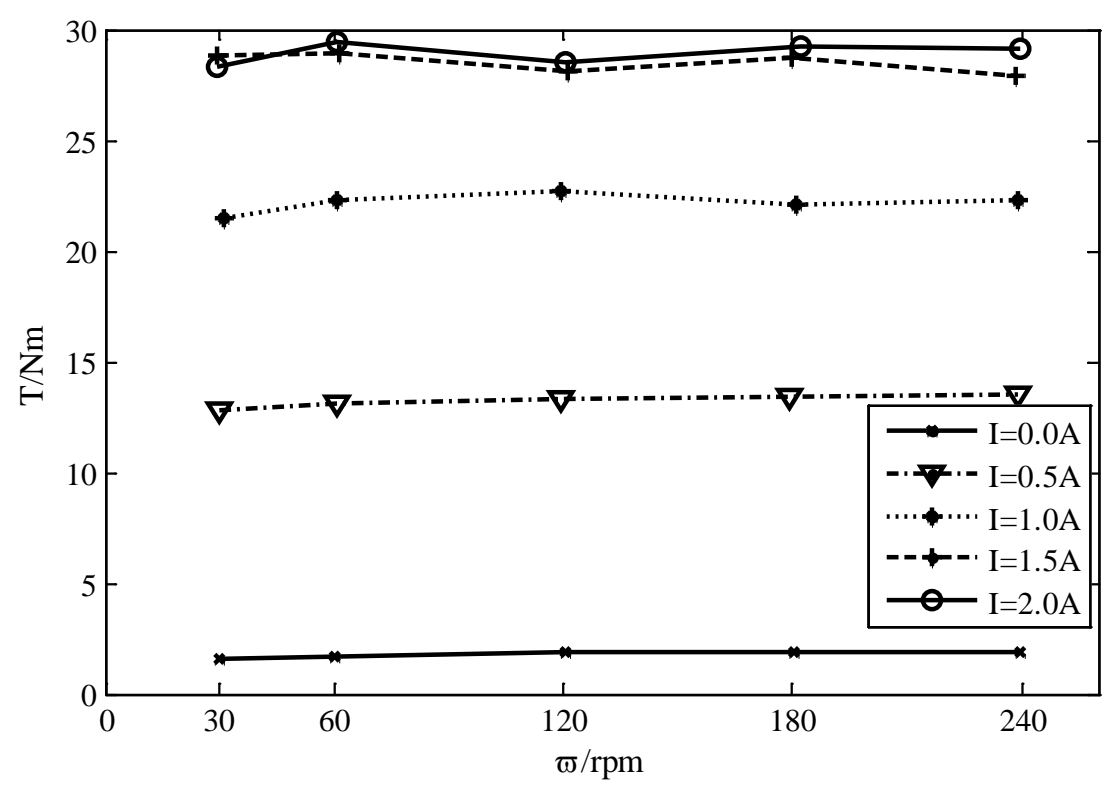

Figure 14. Total transmitted torque vs. rotary speed at different coil currents

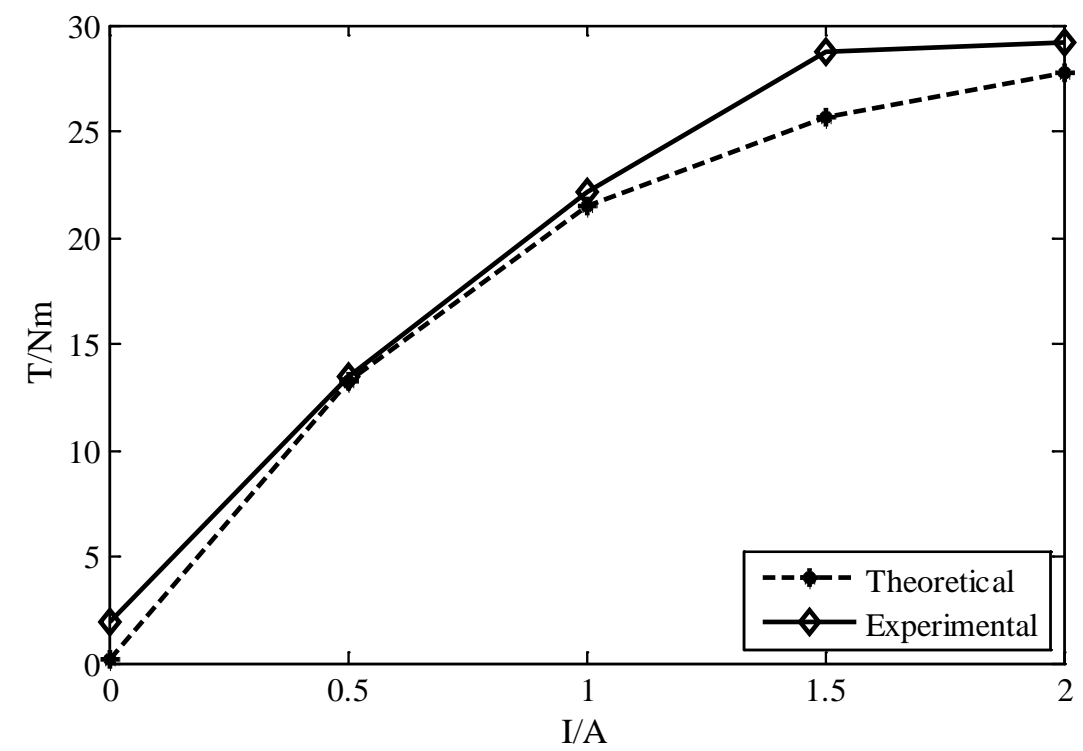




\section{Conclusions}

A preliminary study aimed to develop an intelligent civil structure, equipped with a semi-active structural member, MR pin joint, is proposed in this paper. Both theoretical analyses and experimental studies were conducted in order to investigate the dynamics of the MR pin joint. Based on principles of fluid mechanics, a dynamic model was developed to describe the dynamic behaviour of the MR pin joint. It was found, through theoretical and experimental research, that the proposed MR pin joints, with a diameter of $180 \mathrm{~mm}$, has the capability to produce adjustable torques from less than $1 \mathrm{Nm}$ to $30 \mathrm{Nm}$ by simply altering the coil current. The investigation has confirmed that the designed pin joint can be considered a suitable and promising candidate as a smart member for development of an intelligent structure.

\section{Acknowledgement}

This joint research is supported by two research grants, namely, Research Excellence Grant of University of Technology Sydney and the General Project of National Natural Science Foundation of China (Grant No. 50675106). The authors would like to acknowledge the financial supports from the funding bodies.

\section{References}

[1]. Jolly M R., Bender J W, Carlson J D. Properties and applications of commercial magnetorheological fluids. In: Proceedings of the SPIE 5th International Symposium on Smart Structures and Materials, San Diego, CA, USA, 1998, 262-275.

[2]. Carlson J D, Catanzarite D M, Clair KAS. Commercial magnetorheological fluid devices. International Journal of Modern Physics B, 1996, 10(23/24):2857 - 2865.

[3]. Felt D W, Hagenbuchle M, Liu J, Richard J. Rheology of a magnetorheological Fluid. Journal of Intelligent Material Systems and Structures, 1996, 7(5):589-593.

[4]. Dogruer U, Gordaninejad F, Evrensel C A. A new magneto-rheological fluid damper for high-mobility multi-purpose wheeled vehicle (HMMWV). Journal of Intelligent Material Systems and Structures, 2008, 19(6):641-650.

[5]. Zhao Y, Choi Y T, and Wereley N M. Semi-active damping of ground resonance in helicopters using magnetorheological dampers. Journal of the American Helicopter Society, 2004, 49(4):468-482.

[6]. Yang G Q, Spencer B F, Jung H J and Carlson J D. Dynamic modeling of large-scale magnetorheological dampersystems for civil engineering applications. Journal of Engineering Mechanics, 2004, 130(9):1107-1114.

[7]. Lou W J, Ni Y Q and Ko J M. Modal damping and stepping-switch control of stay cables with magnetorheological fluid dampers. Proc. of SPIE, Reno, Nevada, 2001, 4330:354-365.

[8]. Fujino Y, Soong T T, and Spencer B F, Structural control: basic concepts and applications. Proc. ASCE Structures Congress XIV, Chicago, Illinois, 1996, 1277-1278.

[9]. Widjaja J, Samali B and Li J, The use of displacement threshold for switching frequency strategy for structural vibration mitigation. Journal of Mechanical Science and Technology, 2007, 21:865-869.

[10].Carlson J D. MR fluids and devices in the real world. International Journal of Modern Physics B, 2005, 19(7, 8 \& 9):1463-1470.

[11].Carlson J D, Leroy D F, Holzheimer J C et al. US Patent, 9708 853, 1998-01-08. 